\title{
Modulación inflamatoria en el shock traumático*
}

\author{
Drs. DAVID A. MATEO DE ACOSTA A. ${ }^{1}$, EDGAR BEDOLLA O. ${ }^{1}$, \\ JOHN MAKIPOUR J. ${ }^{1}$, JHONELLE A. WAISSBLUTH G. ${ }^{2}$ \\ Departamento de Cirugía General, Advocate Massonic Medical Center, Chicago Illinois, USA. \\ 2 Universidad Mayor, Escuela de Medicina, Santiago, Chile.
}

\begin{abstract}
Inflammatory modulation in traumatic shock

Hemorrhagic hypovolemic shock secondary to trauma is an important cause of morbidity and mortality worldwide. During the last few years, new concepts have emerged and the guidelines of fluid resuscitation in these patients have been redefined. The concept of hypotensive resuscitation has been established and new colloid solutions based on starch have been manufactured, been hydroxyethyl starch in a balanced electrolytic solution, the most studied and successful one. It has been reported, as well, the positive effects of the pharmacologic modulation of the inflammatory pathways in experimental model subjects submitted to hypovolemic shock. Products such as, ethyl pyruvate and the $\mathrm{Na}+\mathrm{H}+$ type 1 inhibitor, BIIB513, have been Studies only experimentally in rodent models using colloids as the primary resuscitation fluid. The significant improvement in the hemodinamyc, pattern and the cardiac and inflammatory indexes and mediators, has created the basis for their use in clinical trials in the near future. The systemic inflammatory response is an important cause of multiple organ failure that increases the late mortality of patients surviving the initial early phases of hypovolemic traumatic shock and its experimental modulation in rodent models with products such as ethyl pyruvate and BIIB513 has produced excellent in vivo and in vitro results.
\end{abstract}

Key words: Hydroxyethyl stach, NHE-1, BIIB513, Ethyl Pyruvate, hypovolemic shock, trauma.

\section{Resumen}

Universalmente se considera el Shock hipovolémico de origen hemorrágico como una importante causa de morbi-mortalidad. Durante los últimos años se ha redefinido los conceptos de la reanimación con líquidos intravenosos en los pacientes con choque hipovolémico y establecido los conceptos de reanimación hipotensa con el uso de nuevos coloides derivados del almidón, tales como el hidroxietil-almidón en solución electrolítica balanceada (Hextend $\mathbb{R})$. Así mismo, se ha reportado el beneficio que conlleva el uso de modificadores de

\footnotetext{
*Recibido el 23 de Noviembre de 2009 y aceptado para publicación el 13 de Abril de 2010.

Este Trabajo no ha sido apoyado por ninguna institución o fuente económica tipo "Grant".

Este Trabajo no presenta conflicto de intereses.

Los autores no son parte de empresas farmacéuticas ni presentan conflicto de intereses con respecto al tema.

Correspondencia: Dr. David A. Mateo de Acosta A.

Advocate Illinois Massonic Medical Center.

836 W Wellington Ave Room 4807, Chicago, IL 60657, USA.

E-mail: drdavidmateodea@yahoo.com.mx
} 
la cascada inflamatoria en modelos experimentales de sujetos sometidos a choque hipovolémico hemorrágico. Productos como el etil piruvato y la BIIB513, un inhibidor selectivo del intercambiador $\mathrm{Na}+/ \mathrm{H}+$ tipo 1 , han sido estudiados sólo experimentalmente en modelos roedores, empleando coloides como principal elemento de reanimación. Al mejorar el perfil hemodinámico, parámetros cardíacos y niveles de mediadores inflamatorios, estos compuestos constituyen una base cierta para ser incluidos en estudios clínicos en un futuro próximo. La respuesta inflamatoria sistémica está íntimamente implicada en la patogénesis de la Falla Orgánica Múltiple, aumentando la mortalidad tardía de pacientes que sobreviven las etapas tempranas del shock hipovolémico hemorrágico traumático. Su modulación experimental con el etil piruvato o bien la BIIB513 ha dado excelente resultado tanto en modelos experimentales in vivo como in vitro.

Palabras clave: Hidroxietil-almidón, NHE-1, BIIB513, etil piruvato, shock hipovolemico, trauma.

\section{Introducción}

Estadísticas norteamericanas reportan que en traumatizados anualmente ocurren más de cincuenta mil muertes a causa de shock hipovolémico hemorrágico $^{1-3}$. El paciente en choque hipovolémico desarrolla una respuesta inflamatoria generalizada, responsable en parte de esta elevada morbi-mortalidad. ${ }^{4}$ La reanimación hidroelectrolítica, a su vez, inicia una respuesta inflamatoria caracterizada por la elevación, del factor de necrosis tumoral a $\left(\mathrm{TNF}-\alpha^{(1)}\right)$ y otras citokinas inflamatorias, que ocasionan efectos metabólicos deletéreos y cambios intracelulares que afectan la bomba de hidrogeniones, $\mathrm{Na}+/ \mathrm{H}+$ tipo $1\left(\mathrm{NHE}-1^{(2)}\right)$, aumentan la anaerobiosis celular, generan acidosis metabólica y producen liberación de mediadores isquémicos. Ello desemboca en un grave estado, con choque irreversible, disfunción miocárdica, fibrilación ventricular y el cese de la función cardíaca $^{5-7}$. Los líquidos de reanimación utilizados más frecuentemente en el paciente en choque hipovolémico, han sido la solución salina isotónica (SI) y la solución de Ringer Lactato (RL) ${ }^{4}$. Estas, ocasionan un incremento de la carga de Cl-, concentración de hidrogeniones $[\mathrm{H}+]$ y una mayor mortalidad $^{8-10}$.

\section{Evolución de las soluciones en el trauma}

Los cuerpos médicos de las fuerzas militares de EUA, han adoptado un protocolo de reanimación para el shock, con hipotensión permisiva, minimizando la cantidad de líquidos administrados, evitando el fenómeno de re-sangrado, la acidosis metabólica y la coagulopatía secundaria. Estas nuevas estrategias incorporan el hidroxietil-almidón al $6 \%$ en solución electrolítica amortiguada con lactato (Hextend ${ }^{\circledR}$ ). Varios grupos han comparado la com- posición de esta solución con el plasma sanguíneo, la solución Salina Isotónica y la solución de Ringer Lactato $^{11}$. Hextend ${ }^{\circledR}$, muestra una concentración de $\mathrm{Na}+, \mathrm{Mg}+$ y $\mathrm{Ca}+2$ cercana a la del plasma. El uso de esta nueva estrategia ha disminuido la tasa de mortalidad, ${ }^{12}$ falla orgánica múltiple (FOM), acidosis metabólica, coagulopatía y disfunción plaquetaria ${ }^{13,14}$ por lo que en el año 2005 , el Hextend ${ }^{\circledR}$ se convirtió en el líquido de reanimación empleado por las Fuerzas Especiales y los Cuerpos Médicos Militares de EUA ${ }^{15}$.

\section{Resucitación farmacológica}

Como hemos mencionado, en el paciente con shock hipovolémico, la respuesta inflamatoria generalizada contribuye a generar la morbi mortalidad. Varios compuestos antiinflamatorios han sido estudiados como coadyuvantes al proceso de reanimación hidroelectrolítica. Uno de los primeros intentos fue el empleo de piruvato (2-acido cetopropanoico). Recientemente, se han estudiado compuestos que inhiben directamente a la NHE-1, tales como la cariporida, (HOE642) y la benzamida-metanesulfonato de N-(aminoiminometil) - 4 - [4-(2-furanilcarbonil) - 1 - piperazinil] - 3 - (metylsulfonil) (BIIB513). La BIIBP513, es el inhibidor más potente y selectivo conocido de la NHE-1 celular. A continuación, analizaremos los estudios que han abierto las puertas a los conceptos de reanimación farmacológica, enfatizando el uso de etilpiruvato (EP) y del BIIB513.

\section{Papel de los derivados del piruvato en la reanimación farmacológica}

El piruvato es un compuesto esencial en las vías del metabolismo intermediario, in vitro posee efectos antioxidantes y anti-citotóxicos. Desafortunadamente, es un compuesto inestable y su combinación

(1) Por sus siglas en inglés Tumoral Necrosis Factor (TNF).

(2) NHE-1 por sus siglas en inglés - $\mathrm{Na} / \mathrm{H}$ Exchanger type-1. 
en una solución acuosa lo convierte en parapiruvato (2,4-dihidroxi-2-metillglutarato), que es un tóxico mitocondrial ${ }^{11,16}$.

El etilpiruvato (EP), posee mejor estabilidad al entrar en contacto con el plasma humano y mantiene las propiedades antiinflamatorias del piruvato ${ }^{17}$, mejorando la sobrevida de los sujetos en modelos experimentales de choque hemorrágico y séptico ${ }^{18,19}$. Este compuesto, disminuye los niveles sistémicos de la proteína de alta movilidad-1 $\left(\mathrm{HMGB}^{(3)}\right)$ y los niveles cardíacos y esplénicos de TNF- $\alpha$.

El EP, en modelos de roedor, tiene efectos positivos en la sobrevida en las 4 primeras horas posteriores al evento traumático. Así mismo, en modelos animales de shock hemorrágico de más del 75\% del volumen sanguíneo calculado, el tratamiento farmacológico coadyuvante a la reanimación, con una dosis única, de $50 \mathrm{mM}$ de EP, aumenta la sobrevida en las primeras 6 hrs post hemorragia, del 11\% al $100 \%$, y disminuye los niveles hepáticos y cardíacos de TNF- $\alpha^{21}$. Agregado a ello se ha observado, que en controles de esplenectomizados, sometidos al mismo protocolo de hemorragia controlada y a las mismas estrategias de reanimación, los niveles de TNF- $\alpha$ son menores cuando los sujetos son tratados con EP, mostrando una respuesta antiinflamatoria menor que los sujetos no esplenectomizados, presumiblemente secundario a la carencia de la regulación esplénica sobre la producción hepática de TNF- $\alpha$, a través de la liberación del factor de transformación $\beta$ (TGF) en la circulación portal ${ }^{20}$.

La proteína HMGB1 se eleva en el paciente con shock hipovolémico hemorrágico al escapar de las células necróticas 2 hrs post-hemorragia. Esta proteína actúa como marcador de necrosis tisular ${ }^{21,22} \mathrm{y}$ mediador inflamatorio, causando entre otros efectos negativos: fibrilación, paro cardíaco ${ }^{4}$ y hemorragia pulmonar $^{23}$. En modelos de choque hipovolémico, los niveles de la proteína HMGB1 disminuyen en aquellos sujetos tratados con EP, no así en los sujetos reanimados sin $\mathrm{EP}^{24}$

\section{Papel de los inhibidores de la Bomba $\mathrm{Na}+/ \mathrm{H}+$ - 1 en la Reanimación Farmacológica}

La hemorragia aguda del shock hipovolémico causa isquemia miocárdica, fibrilación ventricular y paro cardíaco, que es secundario a la acumulación intracelular de $\mathrm{Na}+\mathrm{y} \mathrm{Ca}^{2}$ y a la activación de la bomba $\mathrm{Na}+\mathrm{H}+$ tipo 1 (NHE-1). La inhibición de la NHE-1 disminuye la incidencia de estos eventos cardíacos y la mortalidad del paciente en shock hipovolémico hemorrágico ${ }^{25,26}$.
La BIIB513, es un inhibidor directo de la NHE-1. En modelos porcinos de hipovolemia controlada, la administración previa de BIIB513, aproximadamente 15 minutos antes de inducirse el shock hipovolémico, hace que todos los sujetos sobrevivan hasta 8 hrs. mientras que aquellos sujetos que no reciben este esquema de tratamiento no sobreviven más allá de la fase temprana del choque hipovolémico ${ }^{27}$. Protocolos similares, que siguen guías establecidas de hemorragia controlada, han reportado el perfil de los efectos cardiovasculares que produce el uso de BIIB513 durante las diferentes etapas del shock hipovolémico hemorrágico. Este inhibidor de la NHE1, atenúa la descompensación cardíaca, aumenta el aporte de oxígeno al miocardio, retrasa el inicio de la etapa irreversible del shock hipovolémico hemorrágico y previene la muerte temprana.

La hiper-contractura de la fibra miocárdica es la huella histopatológica de la lesión por isquemia de reperfusión observada en dichos pacientes ${ }^{28}$. Esta se manifiesta como una hipertrofia progresiva del ventrículo izquierdo (VI), con una reducción secundaria del lumen de la cavidad. El VI pasa por un proceso de remodelación y pseudo-hipertrofia ${ }^{27,29}$ directamente proporcional a la severidad de la hipovolemia. En estos pacientes, la pared del VI inicia un proceso de hipertrofia concéntrica y remodelación secundaria a la hiper-contractura miocárdica lo cual conduce a la descompensación cardíaca. En estudios de cultivos de cardiomiocitos en hipoxia, se ha visto que el desarrollo de la híper contractura ventricular se correlaciona con un aumento marcado en la concentración intracelular de trifosfato de adenosina $\left(\operatorname{ATP}^{(4)}\right)$ y de $\mathrm{Ca}+^{2}$ 30,31. La administración previa de BIIB513 atenúa estos cambios protegiendo a los cardiomiocitos de la alteración ${ }^{32}$.

El estado de hipoperfusión tisular propio del choque hipovolémico causa liberación de desechos celulares del metabolismo anaerobio, aumento de la fracción extraída de oxígeno, disminución de la entrega de oxígeno y aumento de su demanda miocárdica debido al incremento de la frecuencia cardíaca. El pre-tratamiento con BIIB513 disminuye indirectamente el consumo de oxígeno al mantener niveles intracelulares adecuados de $\mathrm{Ca}+^{2}{ }^{28}$. Por otra parte, el compromiso de la integridad de la membrana celular, su despolarización permanente ${ }^{33}$, y el edema celular contribuyen al desarrollo de acidosis metabólica e hipercalemia en el paciente choqueado. Durante este proceso las células cardíacas se sobrecargan de $\mathrm{Cl}-, \mathrm{Na}+, \mathrm{y}_{2} \mathrm{O}$, y pierden $\mathrm{K}+10$ cual termina en un estado de disfunción miocárdica

\footnotetext{
(3) Por sus siglas en inglés - high motility protein-1.

(4) Por sus siglas en inglés - adenosine troposphere - trifosfato de adenosina.
} 
y el paro cardíaco ${ }^{34}$. El pre-tratamiento con BIIB513 atenúa la acidosis metabólica y aumenta el tiempo entre el inicio de la hemorragia y el desarrollo de acidosis metabólica ${ }^{28}$.

Consecuentemente, en el shock hipovolémico, el apoyo farmacológico con la administración de BIIB513, posee efectos cardio-protectores. La adición de una dosis única de BIIB513 (BIIB513du) a los protocolos actuales de reanimación con coloide, en modelos de roedores con choque hipovolémico hemorrágico aumenta en un $38 \%$ la sobrevida a 6 horas. Con respecto a los niveles de mediadores inflamatorios, la administración de una dosis única de BIIB513, disminuye significativamente las concentraciones de TNF- $\alpha$, molécula de adhesión intracelular - $1\left(\right.$ ICAM- $\left.^{(5)}\right)$ y proteína $\mathrm{C}$ reactiva $\mathrm{C}$ (PCR) observadas en los sujetos que son reanimados con coloides. En ellos, se observa también una infiltración hepática significativa de neutrófilos y aumento de la actividad de mieloperoxidasa (MPO) hepática ${ }^{35}$.

Los niveles de las enzimas hepáticas ALT y AST, marcadores de lesión hepática, incrementan en 2,9 veces y en 2,8 veces respectivamente en sujetos en shock hipovolémico hemorrágico. El tratamiento con una dosis única de BIIB513 reduce los niveles de ALT en $41 \%$ y de AST en $16 \%$. Con respecto a la lesión renal, el monitoreo de los niveles séricos de urea ha mostrado que en estos sujetos, existe un aumento de los niveles séricos de urea del 144\% a las $24 \mathrm{hrs}$ del evento hemorrágico, mientras que en aquellos sujetos tratados con una dosis única de BIIB513, se reducen los niveles de urea en un 23\% con respecto a los no tratados.

\section{Conclusiones}

La respuesta inflamatoria sistémica se implica intimamente en la patogénesis de la Falla Orgánica Múltiple, aumentando la mortalidad tardía de los pacientes que sobreviven las etapas tempranas del shock hipovolémico hemorrágico traumático. Esta respuesta inflamatoria se caracteriza por la regulación al alza de las citokinas pro inflamatorias, produciendo la lesión pulmonar, intestinal, hepática y renal mediada por neutrófilos. El Etil Piruvato actúa como un modulador de la respuesta inflamatoria en modelos animales de shock hipovolémico hemorrágico y su uso en dosis única, previo a la reanimación, incrementa la sobrevida de los sujetos en las fases tempranas y tardías del shock. Su uso también disminuye los niveles hepáticos y cardíacos de TNF- $\alpha$ en modelos experimentales.

La proteína HMGB1 es un mediador inflamatorio que causa paro cardíaco, daño pulmonar agudo, y exacerba la activación de la cascada inflamatoria en el paciente en shock hemorrágico. Esta se libera en el torrente circulatorio en las primeras $2 \mathrm{hrs}$ que siguen al trauma, actuando como un marcador de necrosis celular. Los niveles de esta proteína disminuyen significativamente con el uso de EP.

La respuesta inflamatoria, resultado del evento hipovolémico hemorrágico, ocasiona también descompensación y colapso cardíaco, secundario a una sobrecarga de calcio intracelular del cardiomiocito y a la hiperactividad del NHE-1. Estos cambios, se manifiestan a través de la híper contractura del ventrículo izquierdo, la disminución del aporte de oxígeno e incremento de la demanda de este al miocardio. La reanimación con soluciones cristaloides lava el $\mathrm{pH}$ ácido del líquido extracelular y reactiva al NHE-1 para restaurar el pH intracelular ocasionando una sobrecarga de $\mathrm{Ca}^{2}{ }^{2}$ y disfunción miocárdica hasta su arresto. La administración del EP y BIIB513, incrementa la sobrevida y disminuye los niveles séricos de los mediadores inflamatorios, así como los marcadores de lesión hepática y renal.

\section{Referencias}

1. Anderson RN, Smith BL. Deaths: Leading causes for 2002. Natl Vital Stat Rep 2005; 53: 1-89.

2. Acosta JA, Yang JC, Winchell RJ, Simons RK, Fortlage DA, Hollingsworth-Fridlund P. Lethal injuries and time to death in a level I trauma center. J Am Coll Surg 1998; 186: 528-533.

3. Sauaia A, Moore FA, Moore EE, Moser KS, Brennan R, Read RA, et al. Epidemiology of trauma deaths: A reassessment. J Trauma 1995; 38: 185-193.

4. Ulloa L, Tracey KJ. The "cytokine profile": A code for sepsis. Trends Mol Med 2005; 11: 56-63.

5. Tracey KJ, Cerami A. Tumor necrosis factor, other cytokines and disease. Annu Rev Cell Biol 1932; 39: 317-343.

6. Vaagenes P, Ginsberg M, Ebmeyer U, Ernster L, Fischer M, Gisvold SE, et al. Cerebral resuscitation from cardiac arrest: pathophysiologic mechanisms. Crit Care Med 1996; 24; 2 (Suppl): S57-S68.

7. Hartmann AF, Senn MJ. Studies in the metabolism of sodium r-lactate. III Response of human subjects with liver damage, disturbed water and mineral balance, and renal insufficiency to the intravenous injection of sodium r-lactate. J Clin Invest 1932; 11: 345-355.

(5) Por sus siglas en ingles Intracellular Adhesion Molecule - 1. 
8. Kellum JA, Bellomo R, Kramer DJ. Etiology of metabolic acidosis during saline resuscitation in endotoxemia. Shock 1998; 9: 364-368.

9. Scheingraber S, Rehm M, Sehmisch C. Rapid saline infusion produces hyperchloremic acidosis in patients undergoing gynecologic surgery. Anesthesiology 1999;90: 1265-1270.

10. Stacpoole PW, Wright EC, Baumgartner TG: Natural history and course of acquired lactic acidosis in adults. DCA-Lactic Acidosis Study Group. Am J Med 1994; 97: 47-54.

11. Kellum JA. Fluid resuscitation and hyperchloremic acidosis in experimental sepsis: Improved short-term survival and acid-base balance with Hextend compared with saline. Critical Care Medicine 2003; 30: 157-164.

12. Montgomery CM, Fairhurst AS, Webb JL. Metabolic studies on heart mitochondria. III. The action of parapyruvate on alpha-ketoglutaric oxidase. J Biol Chem 1956; 221: 369-376.

13. Baskett TF. The resuscitation greats: Sydney Ringer and lactated Ringer's solution. Resuscitation 2003; 58: 5-7.

14. Nielsen VG, Tan S, Brix AE, Baird MS, Parks D. Hextend (hetastarch solution) decreases multiple organ injury and xanthine oxidase release after hepatoenteric ischemia -reperfusion in rabbits. Critical Care Med 1997; 25: 1565-1574.

15. United States Army Medical Department Center and School Combat Lifesaver Course: Student Self-Study, IS0871, Progressive Management Publishers "A" edition, 25 Julio 2005. pp 23-48.

16. Vonkorff RW. Pyruvate-C14, purity and stability. Anal Biochem 1964; 8: 171-178.

17. Ulloa L, Ochani M, Yang H, Tanovic M, Halperin D, Yang R, et al: Ethyl private prevents lethality in mice with established lethal sepsis and systemic inflammation. Proc Natl Acad Sci USA 2002; 99: 12351-12356.

18. Tawadrous ZS, Delude RL, Fink MP. Resuscitation from hemorrhagic shock with Ringer's ethyl pyruvate solution improves survival and ameliorates intestinal mucosal hyperpermeability in rats. Shock 2002; 17: 473-477.

19. Yang R, Gallo DJ, Baust JJ, Uchiyama T, Watkins SK, Delude RL, et al. Ethyl pyruvate modulates inflammatory gene expression in mice subjected to hemorrhagic shock. Am J Physiol Gastrointest Liver Physiol 2002; 283: 212-221.

20. Macias CA, Chiao JW, Harada T, Levy MM. Small volume resuscitation with a solution containing ethyl pyruvate improves survival in a lethal model of hemorrhagic shock. Crit Care Med 2005; 33: 32-34.

21. Ombrellino M, Wang H, Ajemian MS, Talhouk A, Scher LA, Friedman SG, et al. Increased serum concentrations of highmobility- group protein 1 in haemorrhagic Shock. Lancet 199; 354: 1446-1447.

22. Ulloa L, Messmer D. High-mobility group box 1
(HMGB1) protein: Friend and foe. Cytokine Growth Factor Rev 1999; 17: 189-201.

23. Abraham E, Arcaroli J, Carmody A, Wang H, Tracey KJ: HMG-1 as a mediator of acute lung inflammation. $\mathrm{J}$ Immunol 2000; 165: 2950-2954.

24. Cai B, Chen G, Lin X, Miller E, Szabo C, Edwin D, Lius U. Anti-inflammatory adjuvant in resuscitation fluids improves survival in hemorrhage. Crit Care Med 2009; $37: 3$.

25. Anderson SE, Murphy E, Steenbergen C, London RE, Cala PM. Na $+/ \mathrm{H}+$ exchange in myocardium: effects of hypoxia and acidification on $\mathrm{Na}$ and $\mathrm{Ca}$. Am J Physiol 1990; 259: C940-C948.

26. Wu D, Stassen JM, Seidler R, Doods H. Effects of BIIB513 on ischemia induced arrhythmias and myocardial infarction in anesthetized rats. Basic Res Cardiol 2000; 95: 449-456.

27. Wu D, Arias J, Bassuk J, Doods H, Seidler R, Adams JA, et al. Na/Hexchange inhibition delays the onset of hypovolemic circulatory shock in pigs. Shock 2008; 29: 519-525.

28. Korge P, Goldhaber JI, Weiss JN. Phenylarsine oxide induces mitochondrial permeability transition, hypercontracture, and cardiac cell death. Am J Physiol Heart Circ Physiol 2001; 280: 2203-2213.

29. Di Segni E, Preisman S, Ohad DG, Battier A, Boyko V, Kaplinsky E, et al. Echocardiographic left ventricular remodeling and pseudohypertrophy as markers of hypovolemia. An experimental study on bleeding and volume repletion. J Am Soc Echocardiogr 1997; 10: 926-936.

30. Henden T, Aasum E, Folkow L, Mjos OD, Lathrop DA, Larsen TS. Endogenous glycogen prevents $\mathrm{Ca} 2+$ overload and hypercontracture in harp seal myocardial cells during simulated ischemia. J Mol Cell Cardiol 2004; 37 : 43-50.

31. van Borren MM, Baartscheer A, Wilders R, Ravesloot JH. NHE-1 and NBC during pseudo-ischemia/reperfusion in rabbit ventricular myocytes. J Mol Cell Cardiol 2004; 37: 567-577.

32. Gumina RJ, Auchampach J, Wang R, Buerger E, Eickmeier $\mathrm{C}$, Moore $\mathrm{J}$, et al. $\mathrm{Na}(+) / \mathrm{H}(+)$ exchange inhibition induced cardioprotection in dogs: effects on neutrophils versus cardiomyocytes. Am J Physiol Heart Circ Physiol 2000; 279: 1563-1570.

33. Shires GT, Cunningham JN, Backer CR, Reeder SF, Illner $\mathrm{H}$, et al. Alterations in cellular membrane function during hemorrhagic shock in primates. Ann Surg 1972; 176: 288-295.

34. Illner HP, Cunningham JN Jr, Shires GT. Red blood cell sodium content and permeability changes in hemorrhagic shock. Am J Surg 1982; 143: 349-355.

35. Wu D, Dai H, Arias J, Latta L, Abraham W. Lowvolume resuscitation from traumatic hemorrhagic shock with $\mathrm{Na} / \mathrm{H}$ exchanger inhibitor Crit Care Med 2009; 37 : 329-335. 